\title{
RESIDUAL FINITENESS AND 'FREE' DISTRIBUTIVELY GENERATED NEAR-RINGS
}

\author{
DAVID J. JOHN \\ (Received 15 January; revised 9 April 1979) \\ Communicated by $\mathbf{H}$. Lausch
}

\begin{abstract}
Let $\mathbf{V}$ be a variety of groups in which the free group is residually finite, and let $S$ be a residually finite semigroup. Let $N_{\mathrm{v}}(S)$ be the 'free' distributively generated near-ring constructed from $S$ and V. Theorem; $N_{v}(S)$ is residually finite.
\end{abstract}

1980 Mathematics subject classification (Amer. Math. Soc.): 16 A 76.

A near-ring $N$ is a set with two binary operations + and $\cdot$, such that $\{N,+\}$ is a group, $\{N, \cdot\}$ is a semigroup, and $\cdot$ is left distributive over + . A distributively generated near-ring is a near-ring $N$ which is additively generated by a set of right (and left) distributive elements.

Given a variety $\mathbf{V}$ of groups and a semigroup $S$ we define a new distributively generated near-ring, $N_{\mathbf{v}}(S) .\left\{N_{\mathbf{v}}(S),+\right\}$ is the free group in $\mathbf{V}$ on free generators of $S$. We define $\left\{N_{\mathrm{v}}(S), \cdot\right\}$ inductively on the words of $\left\{N_{\mathrm{v}}(S),+\right\}$. If $s, t \in S$ and $u, v, w \in N_{\mathrm{v}}(S)$, then $s \cdot t=s t$ (the product of $s$ and $t$ in $S$ ), and

$$
\begin{array}{r}
(-u) \cdot t=-(u \cdot t), \text { and }(w+u) \cdot s=w \cdot s+u \cdot s, \text { and } w \cdot(u+v)=w \cdot u+w \cdot v, \\
\text { and } w \cdot(-u)=-(w \cdot u), \text { and } w \cdot 0=0 .
\end{array}
$$

It has been shown in Fröhlich (1960) and Evans and Neff (1964) that

$$
\left\{N_{\mathrm{v}}(S),+, \cdot\right\}
$$

is a distributively generated near-ring. When $\mathbf{V}$ is the variety of all groups and $S$ is the free semi-group in the variety of all semigroups, $N_{\mathrm{v}}(S)$ is the 'free' distributively generated near-ring.

A near-ring $N$ is residually finite if for any $n \neq 0$ belonging to $N$ there exists a finite near-ring $N_{f}$ and a near-ring homomorphism $\theta: N \rightarrow N_{f}$ such that $n \theta \neq 0$. 
More generally, an algebra $A$ is residually finite if for any distinct $u, v \in A$, there exists a finite algebra $F$ in the variety generated by $A$ and a homomorphism $\beta: A \rightarrow F$ such that $u \beta \neq v \beta$.

We will show that if $\mathbf{V}$ is a variety of groups in which all free groups are residually finite, and if $S$ is a residually finite semigroup, then $N_{\mathbf{v}}(S)$ is a residually finite distributively generated near-ring.

THEOREM. If $\mathrm{V}$ is a variety of groups in which all free groups are residually finite, and if $S$ is a residually finite semigroup, then $N_{\mathrm{v}}(S)$ is residually finite.

Proof. Let $w_{0} \in N_{\mathrm{v}}(S), w_{0}$ different from zero. We can write $w_{0}$ as an additive word in terms of distinct $s_{i} \in S$, say $w_{0}\left(s_{1}, \ldots, s_{n}\right)$. Since $\left\{N_{\mathrm{v}}(S),+\right\}$ is a free group in $\mathbf{V}$, and by hypothesis a free group $\mathbf{V}$ is residually finite, there exists a finite group $G$ in $\mathrm{V}$ and elements $x_{1}, \ldots, x_{n}$ of $G$ such that $w_{0}\left(x_{1}, \ldots, x_{n}\right)$ is not zero. Let $\mathrm{V}(G)$ be the variety generated by $G$.

Since $S$ is residually finite, for any $s_{i}, s_{j} \in S, i \neq j$, there exists a finite semigroup $R_{i j}$ and a homomorphism $f_{i j}: S \rightarrow R_{i j}$ such that $s_{i} f_{i j} \neq s_{j} f_{i j}$. Let $R=\prod_{i \neq j} R_{i j}$, and define $f: S \rightarrow \prod_{i \neq j} R_{i j}$ by $t f=\left(t f_{i j}\right) . f$ is a homomorphism and $s_{i} f \neq s_{j} f$ for $i \neq j$. Suppose $R$ contains $m$ elements, $r_{1}, \ldots, r_{m}$.

Construct $N_{\mathbf{v}(G)}(R)$, the distributively generated near-ring with distributive generating set $R$ such that $\left\{N_{\mathbf{v}_{(G)}}(R),+\right\}$ is free in $\mathbf{V}(G)$ on generators $R$. Since $G$ is a finite group and $\left\{N_{\mathbf{v}(G)}(R),+\right\}$ is a finitely generated free group in $V(G)$, $\left\{N_{\mathbf{v}_{(G)}}(R),+\right\}$ is finite. Thus $\left\{N_{\mathrm{V}(G)}(R),+, \cdot\right\}$ is a finite distributively generated near-ring. Let $\theta: N_{\mathrm{v}}(S) \rightarrow N_{\mathrm{v}(G)}(R)$ be the near-ring homomorphism determined by

$$
g_{i} \theta= \begin{cases}g_{i} f & \text { if } g_{i} \in\left\{s_{1}, \ldots, s_{n}\right\} \\ 0 & \text { otherwise. }\end{cases}
$$

Now we have $w_{0}\left(s_{1}, \ldots, s_{n}\right) \theta=w_{0}\left(s_{1} \theta, \ldots, s_{n} \theta\right)=w_{0}\left(s_{1} f, \ldots, s_{n} f\right) . f$ was chosen so that $s_{i} f \neq s_{j} f$ for $i \neq j$, so $\left\{s_{1} f, \ldots, s_{n} f\right\}$ is a set of $n$ distinct elements of $R$, that is $n$ distinct free generators of $\left\{N_{\mathbf{v}(G)}(R),+\right\}$. $G$ was chosen with the restriction that $w_{0}\left(x_{1}, \ldots, x_{n}\right)$ was not zero. Since $N_{\mathrm{v}(G)}(R)$ is free in $\mathrm{V}(G)$ the map $s_{i} \rightarrow x_{i}$ can be extended to a homomorphism, hence $w_{0}\left(s_{1} f, \ldots, s_{n} f\right)$ is not zero.

We now have that $N_{\mathbf{v}}(S)$ is residually finite.

In John and Neff (1979) it is proved that the free near-ring $N_{0}$ in $\mathscr{N}_{0}$, the variety of near-rings with the additional identity $0 x=0$, is a subnear-ring of the 'free' distributively-generated near-ring. Hence, we have that $N_{0}$ is residually finite.

Portions of this paper appear in the author's Ph.D. dissertation written under the direction of Professor Mary F. Neff at Emory University. 


\section{References}

T. Evans and M. F. Neff (1964), 'Substitution algebras and near-rings I', Notices Amer. Math. Soc. 11, 757.

A. Fröhlich (1960), 'On groups over a d.g. near-ring I. Sum constructions and free $R$-groups', Quart. J. Math. Oxford (Ser. II) 193-210.

David J. John and Mary F. Neff (1979), 'The word problem is solvable in $N_{0}{ }^{\circ}$, Notices Amer. Math. Soc. 26, A-45.

J. D. P. Meldrum (1976), 'The group distributively generated near-ring', Proc. London Math. Soc. (3), 32, 323-346.

Günter Pilz (1976), Near-rings (North-Holland, Amsterdam).

Department of Mathematics and Computer Science

Valdosta State College

Valdosta, Georgia 31601

U.S.A. 